\title{
Generative Music with the Living Machine: Using Rule-Based Improvisation to Generate Narrative and Soundtrack
}

\section{Ryan Martin}

\section{Introduction}

In this paper, I propose the term generative improvisation to describe improvisations that are constrained by an explicitly human-designed framework as opposed to one that has developed over time with relation to one or more musical styles, what I refer to as idiomatic improvisation. I will demonstrate the role constraints play in this form of improvisation and use this insight to explain how rule-based improvisation can be used to generate the live performance of a coherent narrative and soundtrack. A coherent narrative and soundtrack, for the purposes of this paper, is a situation where the pairing of a narrative-conveying medium (such as video or narration) and a soundtrack clearly communicate meaningful narrative information according to the conventions of Hollywood and Hollywood-inspired films and media. To achieve this goal, I propose basing the constraints of the improvisation on works that already facilitate a coherent relationship between sound and narrative, specifically narrative film and video games. The work accompanying this paper, Narrative Generator (for Living Machine) (Martin), is an example of this kind of generative improvisation and will be used throughout the paper to show how the proposed ideas can be applied. The score for Narrative Generator (for Living Machine) and a recording of a performance are available as supplemental downloads from Critical Studies in Improvisation IEtudes critiques en improvisation.

\section{Generative Improvisation}

Musical improvisation occurs in cultures throughout the world and takes many different forms. Bruno Nettl defines musical improvisation as "the creation of a musical work or the final form of a musical work, as it is being performed" (1). This definition of improvisation is problematic, however, because even pre-composed works leave certain aspects undefined (such as tone, rubato, articulation), meaning that all musical performances inevitably feature some level of creation during performance. Nevertheless, the idea that the music is created mid-performance is integral to the western definition of improvisation. This improvised material, however, does not come from nowhere. The reason improvisers are able to generate music mid-performance is by employing a framework that constrains their musical output (Nettl 1; Pressing 51). These frameworks function by defining certain musical understandings for the improviser, such as the expectation that jazz musicians improvise in relation to the harmonies over which they are playing (Berliner 198) or by limiting what the performer can do, such as constraining the performer to a certain key or mode. When improvising freely, performers are not limited by a specific set of expectations or the confines of a piece of music and are therefore limited only by their physical capabilities with the instrument and their personal musical identity (Bailey 83). Generative improvisation, the term I am proposing in this paper, occurs when an otherwise free improvisation is additionally constrained by a framework consciously devised by a group or an individual. There are various approaches to generative improvisation, including Lawrence "Butch" Morris's Conduction® method, in which different hand gestures have codified meanings that shape the improvisers' contribution (Morris 33), and John Zorn's game pieces, in which a set of rules governs the ways improvisers interact with each other (Brackett 50-54). Importantly, these constraints are not arbitrary, but are adjusted by their creators to produce specific effects, musical or otherwise.

In the case of Zorn's Cobra (1984), the rules, as well as Zorn's specific choice of performers, facilitate a "psychodrama" in which the tension between performers' personalities are 
brought to the fore (Brackett 55-56). This effect is achieved by allowing performers to make hand gestures that temporarily redefine the available musical choices of the whole ensemble, such as the "runner" cue which instructs everyone currently playing to stop and allows the caller of this cue to choose who enters (Brackett 50-54). By allowing performers to limit the freedom of the entire ensemble and making a conscious effort to include performers with various personalities, Zorn creates a specific ensemble dynamic in which some musicians try to seek as much power as they can, whilst others just do what they are told (Bailey 77-78).

Whilst the generation of a specific ensemble dynamic is Zorn's primary intention, the improvisational constraints also determine the possibilities of the musical output. For example, the ability for performers to invoke drastic changes in the texture, instrumentation, and musical style of the ensemble means that performances generally become a series of contrasting sections. "Sensyo," from the album Cobra-Tokyo Operations '94, demonstrates this. In the recording, which lasts six minutes and twelve seconds, there are twenty-one drastic changes in either the texture, instrumentation, or style of the music. Between 0:51 and 3:07, drastic changes in the character of the music occur at five to twenty-second intervals and from 4:45 to 6:06, the music changes every ten to twenty-two seconds. Both the "psychodrama" of the ensemble members and this prevalence of abrupt, contrasting changes in the music, are a direct result of the constraints that Zorn has placed on the improvisation.

It is important to note that the purpose of the term generative improvisation is not to convolute the existing nomenclature or create a needlessly specific category, but to reframe our understanding of these kinds of improvisations. Generative improvisation explains the final musical result of the improvisation as a dialogue between the performers and the human-designed framework restraining them, and this has interesting ramifications for understanding how individual freedom works during these kinds of improvisations. Further, by illuminating the importance of the set of restrictions on the performers, the term highlights another perspective from which we can position these improvisations within the society producing them, and that is how the performer relationships created by the improvisational constraints reflect and interact with social relationships in the real world.

\section{Generating Coherent Narrative and Soundtrack}

Narrative Generator (for Living Machine) is the practical component of the practice-based honours thesis I submitted as part of my undergraduate music degree at the University of New South Wales in Sydney, Australia. I was interested in taking the rule-based methods of Zorn and Morris and applying them in a new way, specifically, combining them with narrative. As a result, Narrative Generator leverages the idea that human-composed constraints can generate specific musical effects and specific ensemble dynamics to develop an improvisation that generates a coherent spoken narrative and live soundtrack. I achieved this goal by appropriating various theories and practices used by filmmakers and game developers to ensure my project had a relationship between the soundtrack and narration at least as coherent as existing narrative-based media.

First, it is necessary to clarify what I mean by "coherent narrative and soundtrack". Consider the well-known "boulder scene" from Raiders of the Lost Ark in which the protagonist, Indiana Jones, runs from a large boulder rolling towards him. Imagine three different soundtracks for this scene: the original soundtrack which features the sound effect of the boulder rolling accompanied by fast, repeated, dissonant chords from the orchestra; the movie's main theme, which is a triumphant medium-tempo march in a major key; and radio static. For viewers that have sufficient experience with films employing the musical conventions of Hollywood (what Anahid Kassabian calls competence), the first soundtrack, when combined with the visuals, will evoke feelings of fear, create tension for the audience, 
and portray Indiana Jones as someone in great danger. The second scenario, however, evokes excitement in the audience and portrays the action on screen as adventurous, but with little real danger for the hero. Both of these options involve the sound and image working in tandem to produce clear narrative meaning to a sufficiently competent audience (Kalinak 16-18). The final proposed soundtrack, radio static, has a different effect. Whilst the visuals more or less concretely portray Indiana Jones running away from a boulder, this example lacks concrete information on the mood, emotions of the character, the pacing of the scene, and various other pieces of information that are usually signified to the viewer when images are accompanied by a soundtrack (Kalinak 1). The first two scenarios demonstrate a coherent narrative and soundtrack: the simultaneous presence of sound and a narrative-conveying medium, the combination of which, when shown to a competent viewer, conveys reasonably clear information on various aspects of the narrative to the degree conventional in Hollywood sound film and the various other forms of media influenced by it.

\section{Claudia Gorbman's Seven Principles}

Claudia Gorbman, in her book Unheard Melodies: Narrative Film Music, proposes seven principles that describe the function of music in classical films, all of which are addressed by Narrative Generator (for Living Machine) in some manner. Whilst Gorbman's theories were explicitly based on the music of classical Hollywood films; they nevertheless apply to soundtracks in general, as well as to conventional films created after that period. Her seven principles are:

1. Invisibility

2. Inaudibility

3. Signifier of Emotion

4. Narrative Cueing

5. Continuity

6. Unity

7. The intentional violation of these principles for a specific effect (Gorbman 73)

Invisibility refers to the way that the apparatus used to create film music, such as the musicians or microphone, are not visible in the images themselves, unless they are part of the diegesis or narrative world (Gorbman 73-75). Inversely, when an object that could logically create a sound is synchronized with that sound, we assume that sound is diegetic, such as Señor Bob playing "Silent Night" on the piano in The Hateful Eight (01:26:11). ${ }^{1}$ Whilst breaking this rule can sometimes be used for comedic purposes, doing so generally shatters the audience's immersion in the world of the narrative (Gorbman 75).

Inaudibility refers to the way that film music generally exists in the background of the viewer's mind, not demanding the focus of their attention, but nevertheless altering the information communicated by the image. The cutting of music to fit the length of the events on screen, the mixing and composing of music in a way that does not overpower the dialogue, beginning and ending music in ways that does not draw attention to itself, and using music that reinforces the mood and movement of the image are all film-making practices that demonstrate the concept of inaudibility (Gorbman 76-78). Like invisibility, inaudibility serves to immerse the audience into the world of the narrative by deemphasizing music and other elements of the soundtrack, such that they are not really "there" in the diegesis of the film.

The third principle of classical film music is the soundtrack's ability to signify emotion (Gorbman 79). Evoking certain emotions in the viewer is important for making the audience empathize with the characters and to further immerse the audience into the world of the narrative (Kalinak 4-5) by making the world portrayed by the images seem more real 
(Cohen 263). This function is important for allowing viewers to experience what Gorbman calls a film's "inner truth" - the deeper meanings beyond what is merely represented by the images (Gorbman 79).

Narrative cueing refers to sound's role in establishing the boundaries and perspectives of the spectator and the ability of sound to shape the meaning of the image (Gorbman 82-84). Some examples of the soundtrack defining boundaries in a film include the opening and ending credits, which outline the borders of the film, or the use of music to accentuate transitions to new locations within the narrative world (Gorbman 82-83). Narrative cueing also refers to sound's role in determining from whose perspective the audience experiences a story (Gorbman 84). For example, in Battleship Potemkin, the soundtrack portrays the massacre of the civilians on the Odessa steps as frantic and terrifying, reflecting the experience of the civilians and causing the audience to interpret the scene from that perspective (00:49:02). Later, the music shifts to slow and mournful, seemingly ignoring the frantic images on screen (00:54:32), shifting the perspective to that of an external observer.

Music in classical film is not there purely to service the narrative; it also exists to provide continuity to the moving image by filling gaps in the dialogue and allowing the film to cut to a new scene without being jarring (Gorbman 89). In addition, the score also gives a single film unity. Music does this primarily through the reoccurrence and transformation of themes and motifs (Gorbman 90-91). These techniques allow otherwise disparate images to feel as if they are part of a single connected work of art.

Gorbman's final principle is that any principle may be violated if it is in service to one of the other principles (Gorbman 73). This is an acknowledgement of the fact that Gorbman's seven principles are not fixed rules that determine how composers create their music, but a theoretical framework derived from her observations on Hollywood conventions.

Gorbman's principles outline the functions of sound in conventional Hollywood cinema. In order for Narrative Generator to achieve its goal of generating coherent narrative and soundtrack, the rules need to take these principles into consideration. For example, the principle of inaudibility informed the choice to have the narrator always clearly audible in any performance (Martin 4). This ensures that the soundtrack, whilst still present and affecting the audience, does not interrupt the primary means with which concrete narrative information is communicated. Another example is the concept of continuity. The rules state that whenever an instrumentalist's character is in the scene, they must be playing (Martin 5). Because there will always be at least one character present once the performance has started, the effect of this rule is that there will always be at least one musician playing. This constancy in the music fills in the gaps as the narrator takes breaths or pauses their narration, similar to how music assists with cuts and other transitions in a Hollywood film (Gorbman 89). There are countless examples of how Gorbman's seven concepts have informed specific rules, as well as the overall aesthetic of Narrative Generator; however, it is not the only piece of film scholarship that was used.

\section{Sonic Gestures as Meaning Makers}

A significant function of sound in film is the way it works together with the images to communicate meaningful narrative information (Kalinak 16-18). Film and video game soundtracks do this by employing physiological (Scherer 245) and culturally-encoded (Kassabian 23) sonic gestures. By transplanting these conventional gestures into Narrative Generator (for Living Machine), the resultant improvised soundtrack can more easily work with the narration to create clear narrative meaning. These codified sonic gestures are formalized by the score as a list of moods, emotions, and events that may occur in the narrative, paired with ways they might conventionally be represented in a film or video game soundtrack (Martin 10-14). 
Most of the sonic gestures employed by composers and sound designers in films and video games leverage the audience's competence. This term, proposed by Anahid Kassabian, describes the ability of film-viewers of a specific culture to understand what certain musical gestures signify through repeated exposure to them (23). Indeed, the consistency across various film music handbooks seems to corroborate this idea. ${ }^{2}$ An example is the use of emphasized sound effects that have been time stretched and treated with reverb to portray slow motion in contemporary films and video games (The Matrix 01:46:30; The Hateful Eight 01:59:14; Max Payne 3). In Narrative Generator, this portrayal of slow motion is formalized as one of the cue cards. These cue cards are played throughout a performance by the audience or by the performers and enact a temporary set of conditions on the performers, causing them to recreate some of these competence-leveraging sonic devices (for example, the slow motion cue card requires the electronics to add reverb to their samples and to slow them down; Martin 18-23). In combination with the list of narrative associations (Martin 1014), the cue cards, through the incorporation of codified sonic gestures, ensure, that for an audience competent in Hollywood and Hollywood-influenced films and video games, the soundtrack will interact with the spoken word in a way that conveys meaningful narrative information.

There is more to a soundtrack than music, however. Because the electronics are in charge of actualising the diegetic sounds of the world depicted by the narration (Martin 7), it is highly beneficial to incorporate the theories and practices of sound design into Narrative Generator's rules. Christian Metz theorizes sounds as autonomous aural objects with various acoustic signifiers that denote different associations to the listener. For example, there are certain sonic qualities that signify to us that a particular sound is "lapping," such as that it is relatively weak, that it is discontinuous, that a single occurrence of the sound is made up of at least two sonic events, and that we identify this sound as being related to liquid (Metz 154). However, whilst these qualities signify "lapping" they do not signify the specific source of the lapping (Metz 155-156). This ability for sound to exist independent of its source, yet still contain particular acoustic signifiers, is what allows sound designers to use crumpled cellophane to evoke the crackling of fire or the screams of a monkey and the crack of a whip played in unison to evoke a fictional, futuristic disc launcher (Mancini 362365). Because there is a limit to what sounds performers can create in a live improvisation, this effect is vital for the portrayal of diegetic sound in an improvised situation. Since the use of an extensive library of very specific sounds would greatly inhibit the performer's ability to react to the improvisations of the other performers, it is more effective to use a small set of sounds that have a diverse range of acoustic signifiers. This way, a single sound effect can be used to portray many of the possible sounds of the diegetic world.

\section{Leitmotifs and Recurring Sound}

Sonic gestures do not always imply meaning through cultural conditioning and physiological processes alone. Sound can also obtain meaning through repeated co-occurrence with an aspect of the narrative. The term leitmotif, initially used to refer to Wagner's use of discrete musical units to represent extramusical meaning (Hacohen and Wagner 445), is used by film music scholars to refer to musical units repeated throughout a film that evoke extramusical meaning (Kalinak 11-12). These units can range from a single motif to an entire section of music (Kalinak 11-12). An example of a leitmotif is the musical material in "Married Life," the composition associated with the character Ellie in the animated film Up. Very early in the film, when the protagonist Carl is still a child, he meets a girl named Ellie. When Carl first sees this girl, the strings and clarinets play arpeggios outlining the harmony of "Married Life" (00:03:45), before the composition appears in full as an arrangement for piano and horn when she introduces herself $(00: 04: 41)$. The next occurrence of the material is when Ellie comes to cheer up the injured Carl in the middle of the night. In this case, the harmony of the theme is played on strings and woodwinds as block chords (00:05:29), referring to the theme, but better fitting the subdued narrative situation. This process continues for the rest 
of the film, playing all or part of the theme whenever Ellie is referred to and transforming the material to suit the current narrative situation.

A similar practice of using themes and leitmotifs to represent characters occurs in Narrative Generator. For example, instrumentalists are required to improvise a theme that evokes the personality, characteristics, and current state of their character. This theme must reoccur during character-defining moments, but transform to evoke their character's current emotional, psychological, or physical state (Martin 5). This implementation of leitmotifs and reoccurring themes allows the audience to contextualize the musical qualities of the theme in relation to the character it is representing, better communicating that theme's narrative meaning.

Leitmotifs, recurring themes, and recurring sounds have a particularly interesting function in video games. In games where the player has some level of control over their character's movement through the game world, recurring diegetic and non-diegetic sounds allow the player to more readily identify characters, locations, game objects, and moods within the virtual space (Collins 130). In Dark Souls III, the player is free to explore a world full of dark fantasy creatures that will attack their character on sight. All of these monsters have specific sound effects for different actions, such as the swiping sound of their weapon, or body moving through the air when attacking, or the vocal cry they make when they are killed. These sounds allow the player to better understand the current state of the enemy and respond accordingly, enhancing their ability to play the game effectively.

Similarly, recurring diegetic and non-diegetic sounds in Narrative Generator allow the audience and performers to better understand the emerging narrative. This interaction between sound and narrative is mediated by the motifs and themes section of Narrative Generator's ruleset. This section instructs performers to relate significant aspects of the narrative to specific sonic gestures. If the narrator returns to a previously mentioned aspect of the narrative (e.g. an object in the story, a character, a narrative theme) then the musicians must also return to the music or sounds they were playing when this narrative concept first appeared (Martin 16-17). The same applies to the narrator; if one of the musicians returns to musical material that has been associated with a part of the narrative, then the narrator must return to that narrative concept. The repeated co-occurrence of a sound and an aspect of the narrative as described above allows the soundtrack to develop specific narrative meaning, enhancing the soundtrack's overall ability to convey meaningful narrative information to the audience.

\section{Balancing Freedom and Order}

So far, I have examined how Narrative Generator (for Living Machine) achieves its goal of facilitating the improvised performance of a coherent narrative soundtrack. When creating the work, however, it was important that control over the direction of the performance was distributed across the ensemble. Whilst most film theorists today understand that music and image work together to create narrative meaning (Kalinak 16-18), in reality, the soundtrack is often made to reinforce the meaning already implied by the images. Narrative Generator subverts this convention by forcing the musicians to react to changes in the narration and the narrator to simultaneously react to changes in the music (Martin 3). Further, different performers are assigned different roles, giving them freedom and authority over their part of the performance (Martin 5-9), as long their choices fit with those made by the rest of the ensemble. This ethos is demonstrated most clearly in the work's name: Narrative Generator (for Living Machine). The second half of the title, Living Machine, is an example of the Deleuzian concept of the assemblage (Livesey 18), and refers to the performers, their performing media, the rules, the performers' understanding of music and narrative, the audience, and any other tangible or intangible object that serves the living machine's new function. As described throughout this paper, this new function is generating coherent 
narrative and soundtrack. In this reading of the work, each performer is seen not as an individual imposing their will, but as a component contributing to the machine's wider function.

Another kind of music that shares this goal of democratic music-making is free improvisation. Free improvisations are often group-based and avoid the use of explicit restrictions on the improvisers. Lacking an established set of stylistic conventions or an explicitly outlined set of rules, the contribution of any individual during a free improvisation is mediated by how it fits within the "aural tapestry" created by the ensemble as a whole (Burrows 6-8). The reason performers are able to understand how their contribution will relate to this collective aural tapestry is because of temporary performance-specific meanings assigned to certain musical gestures and mutually agreed upon understandings of what certain musical gestures mean in different contexts ${ }^{3}$ (Burrows 8-11). These mechanisms allow performers to understand what other individual performers are doing and allow them to react accordingly. Unlike generative improvisations, however, these meanings are constructed mid-performance, are less clearly defined, and do not explicitly restrict the performers' musical choices, but simply determine how they understand their musical contributions and those of their ensemble members. Whilst, through this process, free improvisation distributes authorial control amongst the entire ensemble, it is improbable that this process of building meaning in the moment and drawing from shared musical understandings would facilitate a web of performer relationships as specific as that found in Narrative Generator. Because of this, generative and idiomatic improvisations ${ }^{4}$ can at times result in more freedom for performers, as they allow for certain musical devices that would otherwise be unfeasible during free improvisation. For example, if, during a big band-sized free improvisation, some of the performers want to return to previous material at a specific time in an ensemble-wide, coordinated manner, then proceed to all play harmonics, there are few means of achieving this, short of shouting out instructions to the rest of the ensemble. In Morris's Conduction® method though, the conductor does this by pointing to their forehead and placing a certain number of fingers up to capture the material and then later raising their hand with that same number of fingers up to return to it (Morris 83). To have everyone then play harmonics, the conductor would stretch their arms wide and then place their left hand in an "o" shape at chest level (Morris 53,141). Conduction $\circledast$ has its own limitations, of course. One cannot signify a return to previous material if they did not assign it a number as it was happening, and the conductor cannot convey instructions that are not assigned a hand signal within the pre-established lexicon, but that is the point of generative improvisation: it is not limitless. Generative improvisation applies limits so that performers can improvise in a way that would otherwise be unfeasible. This is not to say that the music created by freely improvised performances are simple or not aesthetically unique, it is just that free improvisation values performer freedom above all else, whilst Narrative Generator and other generative improvisations balance this individual freedom with the ability to perform improvisations that employ specific or novel performer relationships.

In practice, Narrative Generator does not constantly grant all performers the same amount of power. Whilst the work aims to prevent any performer from having control over another, some roles inherently have more power than others in certain contexts. For example, considering that each performance aims to tell a coherent narrative and that music communicates this information more ambiguously than spoken word, it is easier for the musicians to adapt what they are playing to the narrator than for the narrator to bend the storytelling to the soundtrack. Analysing the recording of the performance provided with this paper we see that this results in the narrator directing the story more frequently than any other role. Despite a character's presence in the story being equally controlled by narrator and instrumentalist (Martin 3-4), the narrator in this performance delineated all of the times the characters entered and exited a scene. Further, whilst there are a few examples of the musicians leading the narration (such as the trumpeter playing aggressively and the narrator subsequently escalating the situation with the police at 00:07:42), the musicians are 
undoubtedly basing their contribution on the narration for most of the performance. This foregrounding of the voice may also be a result of music and diegetic sound's sub-ordinance to image or speech in most western narrative-based works (Gorbman 76-78). It should be noted that, if strictly following the rules, the narrator and musicians have an equal say in determining the direction of the narrative, and a particularly assertive instrumentalist could just as easily initiate changes to the story with the narrator having to adapt as best they can. Because of the previously discussed ambiguity of the soundtrack and our culture's overvaluation of dialogue and images, it is easier for the musicians to follow the voice, subtly giving the narrator more control over the direction of the improvisation than the other performers.

\section{The Social Impact}

In her article, "After Relational Aesthetics: Improvised Music, the Social, and (Re)Theorizing the Aesthetic," Born criticizes the writings of Small, Attali, and Schutz by pointing out their over-idealization of the microsociality of the performance (45-46). To summarize, she argues that just because an improvisation creates a certain kind of social organisation during a performance, (i.e. the microsociality), this does not mean that form of organisation will spread to wider society. For example, Attali predicts that Free Jazz heralds a new way of music-making free from institutions and commercialization (Born 45). In reality, though, it is not simply the fact that the musicians had a great deal of freedom when improvising that allowed Free Jazz to impact society. For example, the Association for the Advancement of Creative Musicians (AACM), a Chicago-based organisation which promoted black, experimental music throughout the latter half of the twentieth century, did not successfully combat racist and oppressive institutions simply because they frequently programmed freely improvised music. The organisation challenged and eventually changed these racist social structures in a variety of ways external but related to the aesthetics of the performance. Examples of this include funding themselves instead of obtaining a grant or loan from "whitecontrolled power structures" (Lewis 181), overwhelmingly promoting and programming black musicians, and providing lessons for young, aspiring musicians (Lewis 176-177). The relevance of an artist's actions outside of just performance is why Born emphasizes the importance of music's other modes of social mediation. She specifically identifies four, interconnected planes of social mediation, namely the microsociality of the performance, the music's ability to create and maintain communities, its ability to refract wider social relations, and the way it affects and is affected by wider institutional forces (Born 43).

Given that Narrative Generator (for Living Machine) has only been performed for less than a year and has so far only been exposed to a limited audience, it is difficult to empirically assess the work's impact in the latter three planes, as they go beyond the performance. Nevertheless, it is possible to discuss the potential social implications that this work may have in the future. I specifically want to focus on how the work is influenced by and can potentially influence wider institutional forces.

The institutional forces Narrative Generator is most prominently bound up with are those that reinforce conventional composer-performer-audience relationships. Whilst not true for all kinds of music-making, western music often places the composer at the top, the performers in the middle, and the audience at the bottom in terms of authorial control. For example, a common practice in classical music is for the performer to attempt to manifest "what the composer had intended" through their performance (Butt 74), and, whilst listeners and performers of popular music generally value the expression of the performer's personal emotions more than an expression of the intentions of the composer, copyright law still names the songwriter(s) and lyricist(s) as the owners of the music (Copyright; Copyright in General; FAQs on Copyright). Further, the performances often perceived as having the greatest degree of individuality are those that have been greatly arranged or ornamented by the performer. A common response to a unique cover of a popular song, for example, is to 
say "they made it their own." Often in popular music, the act of composing or arranging grants that individual a greater sense of ownership over that music than performing it does. Clearly, then, copyright law and performance conventions grant composers authority over the music, with performers being perceived as individuals bringing the piece to life, as opposed to owners of the music they are playing.

This hierarchy, however, is significantly muddied when discussing improvised works. Zorn may have devised the system of Cobra, and I may have written the rules of Narrative Generator, but the performers themselves ultimately determine the specifics of the music being played. Despite this, most people would agree that both Zorn and I are the authors of our respective works. This assignment of the rule-maker as the author, I argue, is a result of the concept that music exists as an intangible work, with physical performances simply instantiations of an abstraction which represents the true identity of the music (Steingo 8687; Levinson 6; Treitler 483). This perceived intangibility of a musical work can be seen in the way that Zorn and many other composers, including myself, treat our creations as if they are autonomous: giving them titles, detailing the approximate date when they were composed, and considering performances, even those that sound completely different, to be examples of the same musical work. In addition, as part of the submission process for my thesis, it was necessary to record Narrative Generator as a physical score. Copyright law also requires that works exist in some tangible form, generally either as a score or as an audio recording (Copyright; Copyright in General; FAQs on Copyright). Because of our workcentric understanding of music's ontology and academia and copyright law's assertion that the creator of the score owns the musical work, the composer is still in a hierarchically higher position than the performer in Narrative Generator. This, of course, contradicts the piece's goal of distributing authority across the ensemble.

However, just because the work was made to fit into existing, institutionalized understandings of the composer-performer relationship, does not mean that it cannot also point out the unsuitability of this relationship. In Narrative Generator and many improvised works, the performers have a significant role in determining the outcome of the improvisation, yet, because the work is seen to be a better indicator of the music's identity than the performance itself, the composer is generally acknowledged as the author. This can be seen in the fact that many audio releases of improvised works credit the composer as the primary artist of the record. ${ }^{5}$ This is not to suggest that it is morally wrong to name the rulemaker of the improvisation the author or that we need to abandon the work-concept for generative improvisations. Instead, the point of this analysis is to emphasize the awkwardness with which Narrative Generator and similar works fit into normative composerperformer relationships and to hopefully set a precedent for future works to explore different ways of relating the composer(s) and performer so that authorship is distributed more evenly.

Narrative Generator also allows the audience to shape the final result of a performance. Although it is possible to perform it without audience interaction, the work is generally performed with the audience suggesting a genre or setting, as well as directly intervening into the performance by playing "cue cards" that force the ensemble to recreate various conventional narrative events (Martin 17). Although copyright law (FAQs on Copyright; Copyright in General) would not consider this a significant enough contribution to be considered an author of the music, the final result of the performance will nevertheless be vastly different depending on the choices of the audience members. This subverts the typical role of the audience as passively interacting with the performance and gives them some direct control over the outcome of the performance. By placing some authority over the performance with the audience, Narrative Generator further muddies standard composerperformer-audience relationships. 
I have so far explored some of the potential impacts of Narrative Generator; however, these impacts have the potential to reach further and be more significant because of the work's accessibility. There are countless examples of niche or experimental music being successfully integrated into mainstream films and video games because of the way the music is re-contextualized based on its relationship to the narrative. ${ }^{6}$ This same effect occurs with Narrative Generator, allowing its experimental, rule-based procedures to be exposed to a wide array of audience members in a way that they can enjoy and engage with. Audience feedback so far seems to support this assertion. Audience members, even those who have little or no exposure to rule-based improvisation, reported enjoying the performances and experiencing a higher level of engagement because of the performer-audience interactions. This positive experience is important as it increases the number of people that are likely to return to rule-based performances such as Narrative Generator, which share control of the performance amongst the composer, performers and audience. This, in turn, has the potential to normalize musical performances that subvert typical composer-performeraudience relationships and make more people question the ubiquity of the standard composer-performer-audience hierarchy.

\section{Conclusion}

There are a variety of composers and artists who have used rules and systems in their improvisations. The concept of generative improvisation conceptualizes these rules as devices that constrain the choices of the performers and define/redefine the meanings of different musical gestures, generating specific results. I demonstrate this concept with my work Narrative Generator (for Living Machine), which shows how rules can be used to generate improvised narrative and soundtrack, the interaction of which creates meaningful narrative information. A group improvisation, however, is inherently tied to the musical and social relationships of its performers (Born 41), and the constraints of a generative improvisation affect these relationships. The intended set of relationships for Narrative Generator was one where ensemble members work towards a unified goal with control over the performance distributed relatively evenly across the ensemble. Whilst it achieves this goal to a degree, factors internal and external to the rules unintentionally give certain performers more opportunities to control the direction of the performance in certain situations. Looking beyond the social relations of just the performance, we also see how the work, despite its democratic aims, has been influenced by institutional and cultural forces to fit into a typical composer-performer relationship in which authorship is granted overwhelmingly to the composer.

Nevertheless, the distributed authority that does occur in performance disrupts conventional understandings of the composer and performer, demonstrating the unsuitability of a composer's conventional amount of authorial control for improvised works. Combined with the importance of the audience's contribution and the work's accessibility in general, Narrative Generator (for Living Machine) has the potential to make a larger range of individuals question the appropriateness of the typical top-down composer-performeraudience hierarchy. At the very least, the work can, in combination with existing forms of music-making like free improvisation, open up a space for further works that explore a distributed approach to authority in improvisation. The most significant issues of the work are its unintended benefits towards certain improvisers and its adaptation to the existing model of composer-performer-audience. Perhaps future works that question this structure will develop ways of improvising that more evenly distribute authority, not just during the microsociality of the performance, but at the broader social level as well. 


\section{Notes}

${ }^{1}$ Film timings are formatted as follows hours:minutes:seconds.

${ }^{2}$ See, for example, Meyers; Karlin and Wright; Skinner.

${ }^{3}$ Burrows notes that this second point is only true when performers have a similar background, such as an Indian performer's understanding that beat one of a tala (a predetermined rhythmic cycle) is the appropriate place to end an improvisation.

${ }^{4}$ Idiomatic improvisations in this context refers to improvisations constrained by the conventions of an idiom, such as Jazz or Hindustani music.

${ }^{5}$ In CDs such as Cobra: Tokyo Operations '94 by Zorn, Aus Den Sieben Tagen by Stockhausen, and any audio release of Butch Morris's conduction performances, it is the composer of the rules, not the performers, credited as the primary artist.

${ }^{6}$ Such as the use of heavy metal to represent fast-paced action in the video game $D m C$ : Devil May Cry, the use of extreme dissonance and extended techniques to create tension in most contemporary horror films and video games, or the use of textural composition to create a haunting atmosphere in the TV series Hannibal.

\section{Works Cited}

Bailey, Derek. Improvisation: Its Nature and Practice in Music. Da Capo, 1993.

Battleship Potemkin. Directed by Sergei Eisentstein, Mosfilm, 1925.

Berliner, Paul. Thinking Jazz: The Infinite Art of Improvisation. The U of Chicago P, 1994.

Born, Georgina. "After Relational Aesthetics: Improvised Music, the Social, and $(\mathrm{Re})$ Theorizing the Aesthetic." Improvisation and Social Aesthetics, edited by Georgina Born, Eric Lewis \& Will Straw, Duke UP, 2017, pp. 33-58.

Brackett, John. "Some Notes on John Zorn's Cobra." American Music, vol. 28, no.1, 2010, pp. 44-75.

Burrows, Jared. "Musical Archetypes and Collective Consciousness: Cognitive Distribution and Free Improvisation." Critical Studies in Improvisation / Études critiques en improvisation, vol. 1, no.1, 2004, www.criticalimprov.com/index.php/ csieci/article/view/11/36.

Butt, John. Playing with History: The Historical Approach to Musical Performance. Cambridge UP, 2002.

Cohen, Annabel. "Music as a Source of Emotion in Film." Music and Emotion: Theory and Research, edited by Patrik N. Juslin \& John A. Sloboda, Oxford UP, 2001, pp. 24772.

Collins, Karen. Game Sound. The MIT Press, 2008. 
"Copyright." APRA AMCOS, apraamcos.com.au/music-creators/copyright/. Accessed 20 July 2018.

"Copyright in General." U.S. Copyright Office, www.copyright.gov/help/faq/faqgeneral.html\#mywork. Accessed 20 July 2018.

Dark Souls III. From Software, Bandai Namco Entertainment, 2016.

DmC: Devil May Cry. Ninja Theory, Capcom, 2013.

"FAQs on Copyright." European Union Intellectual Property Office, euipo.europa.eu/ohimportal/en/web/observatory/faqs-on-copyright-el. Accessed 20 July 2018.

Gorbman, Claudia. Unheard Melodies: Narrative Film Music. BFI Publishing, 1987.

Hacohen, Ruth and Naphtali Wagner. "The Communicative Force of Wagner's Leitmotifs: Complimentary Relationships between Their Connotations and Denotations." Music Perception: An Interdisciplinary Journal, vol. 14, no. 4, 1997, pp. 445-75.

Hannibal. Created by Bryan Fuller. Sony Pictures Television, 2015.

Hateful Eight, The. Directed by Quentin Tarantino, performances by Samuel L. Jackson, Kurt Russel, Jennifer Jason Leigh, Walton Goggins, Demián Bichir, Tim Roth, Michael Madsen, Bruce Dern, James Parks, and Channing Tatum, The Weinstein Company, 2015.

Kalinak, Kathryn. Film Music: A Very Short Introduction. Oxford UP, 2010.

Karlin, Fred and Rayburn Wright. On the Track: A Guide to Contemporary Film Scoring. Schirmer Books, 1990.

Kassabian, Anahid. Hearing Film: Tracking Identifications in Contemporary Hollywood Film Music. Routledge, 2001.

Levinson, Jerrold. "What a Musical Work Is." The Journal of Philosophy, vol. 77, no. 1, 1980, pp. 5-28.

Lewis, George E. A Power Stronger Than Itself: The AACM and American Experimental Music. The U of Chicago P, 2008.

Livesey, Graham. "Assemblage." The Deleuze Dictionary, edited by Adrian Parr, Edinburgh UP, 2005, pp. 18-19.

Mancini, Marc. "The Sound Designer." Film Sound: Theory and Practice, edited by Elizabeth Weis \& John Belton, Columbia UP, 1985, pp. 361-8.

Martin, Ryan. Narrative Generator (for Living Machine), 2017. Unpublished.

Matrix, The. Directed by The Wachowskis, performances by Keanu Reeves and Laurence Fishburne, Roadshow Entertainment, 1999. 
Max Payne 3. Rockstar Studios, Rockstar Games, 2012.

Metz, Christian. "Aural Objects." Film Sound: Theory and Practice, edited by Elizabeth Weis \& John Belton, Columbia UP, 1985, pp. 154-61.

Meyers, Randall. Film Music: Fundamentals of the Language. Norway: Ad Notam Gyldendal,1994.

Morris, Lawrence. The Art of Conduction: A Conduction ${ }^{\circledR}$ Workbook. New York: Karma, 2017.

Nettl, Bruno, et al. "Improvisation." Oxford Music Online - Grove Music Online, 20 Jan. 2001, updated 3 Sep. 2014, http://www.oxfordmusiconline.com/grovemusic/ view/10.1093/gmo/9781561592630.001.0001/omo-9781561592630-e0000013738 ? start=1\&pos=9\&q=improvisation\&search=quick. Accessed 9 July 2017.

Pressing, Jeff. "Psychological Constraints on Improvisational Expertise and Communication." In the Course of Performance: Studies in the World of Musical Improvisation, edited by Bruno Nettl \& Melinda Russell, U of Chicago P, 1998, pp. 47-67.

Raiders of the Lost Ark. Directed by Steven Spielberg, performances by Harrison Ford, Karen Allen, Paul Freeman, Ronald Lacey, John Rhys-Davies, and Denholm Elliott, Paramount Pictures, 1981.

Scherer, Klaus R. "Which Emotions Can be Induced by Music? What Are the Underlying Mechanisms? And How Can We Measure Them?" Journal of New Music Research vol. 33, no. 3, 2004, pp. 239-51.

Skinner, Frank. Underscore. Criterion Music, 1960.

Steingo, Gavin. "The Musical Work Reconsidered, In Hindsight." Current Musicology, no. 97, 2014, pp. 81-112.

Stockhausen, Karlheinz. Aus Den Sieben Tagen. Rec. 26-31 August 1969 and 20 August 1972, Discogs, 1993.

Treitler, Leo. "History and the Ontology of the Musical Work." The Journal of Aesthetics and Art Criticism, vol. 51, no. 3, 1993, pp. 483-497.

Up. Directed by Pete Docter, performances by Ed Asner, Christopher Plummer, and Jordan Nagai, Walt Disney Studios Motion Pictures, 2009.

Zorn, John. Cobra. 1984. Unpublished: Exists as oral description.

---. “Sensyo." Cobra-Tokyo Operation '94, disk union, 1994. 